\begin{tabular}{lcr}
\hline \multicolumn{3}{c}{ ANNALES } \\
UNIVERSITATIS MARIAE CURIE-SKŁODOWSKA & \\
& LUBLIN - POLONIA & \\
VOL. LXXI, 1 & SECTIO AA & 2016 \\
\hline
\end{tabular}

\title{
Comparison between surface and volumetric properties of short-chain alcohols and some classical surfactants
}

\author{
Magdalena Bielawska, Anna Zdziennicka and Bronisław Jańczuk ${ }^{*}$ \\ Department of Interfacial Phenomena, Faculty of Chemistry, \\ Maria Curie-Skłodowska University, \\ Maria Curie-Skłodowska Sq. 3,20-031 Lublin, Poland \\ "email: bronislaw.janczuk@poczta.umcs.lublin.pl
}

Measurements of the dynamic surface tension of the aqueous solutions of methanol, ethanol, propanol, CTAB and SDDS at their given concentrations were made. From the obtained results and the literature data it was concluded that the adsorption of short-chain alcohols at the water-air interface is somewhat similar to that of classical surfactants. For that reason the relationship between the Gibbs standard free energy of adsorption of short-chain alcohols and classical surfactants at that interface was established. The correlation between the chemical potential of mixing of alcohols and surfactants was also analysed. This analysis concerned the critical aggregation concentration (CAC) of alcohols and the critical micelle concentration $(\mathrm{CMC})$ of surfactants. The chemical potential of surfactant mixing was calculated from the literature CMC data for the homologous series of alkyl sulfates, alkyl sulfonates, alkyl ammonium chlorides, alkyl trimethylammonium bromides, and alkyl pyridinium bromides. The influence of the hydrophobic chain length of alcohol and surfactant molecules on the Gibbs standard free energy of their adsorption at the water-air interface and their chemical potential of mixing were considered. It appeared that there is a linear dependence between these thermodynamic functions and the number of carbon atoms increased by 1 in the hydrocarbon chains of these compounds. This confirms clearly our conclusion that the behaviour of short-chain alcohols and classical surfactants at the water-air interface and in the bulk phase of aqueous solutions is similar. 


\section{INTRODUCTION}

Short-chain alcohols are common organic additives, applied in surfactant systems as modifiers of surface and interfacial properties of the solution [1]. Alcohol-water mixtures have always been seen as interesting due to their anomalous behaviour such as the existence of a viscositycomposition maximum and decrease of the partial molar volume in comparison to that in the "pure" alcohol state. This behaviour depends on the solution microstructure. The adsorption properties of short-chain alcohols at the water-air interface are known and clearly described [2-5]. The efficiency of their adsorption depends on the number of carbon atoms in the molecule but the effectiveness is the same for all these alcohols. However, despite many papers describing the behaviour of alcohols in the bulk phase [2-22], the problem of existence of alcohol aggregates above their proper concentration has not been clearly explained. Several authors [10-13] stated mainly on the basis of the X-ray Diffraction analysis and molecular dynamics methods that pure short-chain alcohols associate in the form of chains, however, ring structures are also possible. Alcohol molecules can self-associate also in aqueous solutions. For example, Zana and Eljebari [6] claimed on the basis of the fluorescence intensity measurements that alcohols form short-lived aggregates, which resemble classical surfactant micelles in some aspects. Yoshida and Yamaguchi [7] as well as Roney et al [8], analysed low-frequency Raman spectra of alcohols and proved that, apart from methanol, alcohols and water form single-component clusters in the bulk phase of the solution at room temperature. However, Dixit et al. [14] as well as Fidler and Rodger [15] stated that even in water-methanol solutions, the local structure of water is similar to that in pure water. Contrary to these opinions, Alavi et al. [16] asserted that short-chain alcohols form strong hydrogen bonds with water, while Yano [4] reported formation of micelle-like aggregates of short-chain alcohols, which destroy the hydrogen bond network in water.

Desnoyers et al. [17] suggested that methanol and ethanol do not aggregate in water (only modify its structure) and do not form mixed micelles with surfactants. However, the presence of small aggregates of methanol, ethanol and propanol was also confirmed by our studies of the surface tension, density and viscosity of their aqueous solutions [2]. Based on them we stated that even methanol molecules aggregate in the bulk phase and for ethanol and propanol these aggregates resemble small micelles at a proper concentration of the solution. Moreover, our studies 
of the surface tension, density and viscosity of the aqueous solutions of the mixtures of anionic, cationic and nonionic classical surfactants with short-chain alcohols and even the mixtures of these surfactants in the presence of an alcohol have shown that alcohol molecules aggregate at their concentration close to the critical aggregation concentration (CAC) determined in their aqueous solutions in the absence of surfactants [18-22]. The CAC value practically does not depend on the kind of surfactant and composition and concentration of the surfactant mixture.

The behaviour of alcohols and surfactants at the water-air interface and in the bulk phase of their aqueous solutions suggests that there should be some correlations between the surface and volumetric properties of alcohols and classical surfactants. Thus the purpose of our paper was to find the relationship between their adsorption and aggregation in aqueous media.

\section{EXPERIMENTAL}

Cetyltrimethylammonium bromide (CTAB) and sodium dodecylsulfate (SDDS) were purchased from Sigma-Aldrich and used without any further purification. Methanol (99.9\% purity, Sigma-Aldrich) was also used without any further purification. Ethanol (99\% purity, SigmaAldrich) was purified by fractional distillation in the presence of magnesium and iodine as an activator [23] and kept over molecular sieves. Propanol (99.5\% purity, Sigma-Aldrich) was purified by the standard method [23]. For the preparation of the solutions doubly distilled and deionised water (Destamat Bi18E) was used and its purity was controlled by the surface tension measurements. The aqueous solutions of methanol (at the concentration $(C)$ equal to 0.13 and $11.97 \mathrm{M}$ ), ethanol $(C=0.13$ and $6.69 \mathrm{M})$, propanol $(C=0.13$ and $3.21 \mathrm{M}), \mathrm{CTAB}\left(C=1 \cdot 10^{-5}\right.$ and $\left.6 \cdot 10^{-4} \mathrm{M}\right)$ and $\operatorname{SDDS}\left(C=1 \cdot 10^{-5}\right.$ and $\left.6 \cdot 10^{-4} \mathrm{M}\right)$ were made.

The dynamic surface tension $\left(\gamma_{L V}\right)$ measurements of the aqueous solutions of methanol, ethanol, propanol, CTAB and SDDS were made at $293 \mathrm{~K}$ under atmospheric pressure by means of Krüss BP100 Bubble Pressure Tensiometer with the integrated compressor and fully automatic liquid surface detection. The temperature was controlled by a jacketed vessel connected with the thermostatic water bath with the accuracy \pm 0.1 $\mathrm{K}$. The tensiometer was controlled by the LabDesk Software. The capillary was made of hydrophobically coated glass and its inner diameter was equal to $0.235 \mathrm{~mm}$. The capillary was cleaned with ethanol and distilled water before the measurements and its cleanness was controlled 
by the dynamic surface tension measurements of water. The surface age was between $1 \cdot 10^{-2}$ and $2.5 \cdot 10^{2} \mathrm{~s}$.

\section{RESULTS AND DISCUSSION}

As it is commonly known, the critical micelle concentration (CMC) of surfactants (or the critical aggregation concentration (CAC) of shortchain alcohols) depends on the method of its determination and this is rather a narrow range of concentration than a single value [1]. The aqueous solutions of classical surfactants (like CTAB or SDDS) and short-chain alcohols were extensively studied by many authors [1, 24-34]. A large amount of available CMC (CAC) data was based on the surface tension measurements [1, 24-34]. In order to check the correctness of these results, the dynamic surface tension measurements of the aqueous solutions of methanol, ethanol and propanol as well as CTAB and SDDS at a few different concentrations were made (Fig. 1).

The chosen concentrations of alcohols and surfactants corresponded to the unsaturated and saturated monolayers of their molecules at the water-air interface $[2,25]$. The chosen concentration range of surfactants and alcohols was different due to their different surface activity. If the same concentrations were used for alcohols as for surfactants, only slight surface tension changes would be observed.

It appeared that the dynamic surface tension values obtained at equilibrium are close to the literature data determined from the surface tension measurements by the du Noüy ring method $[2,25]$. Therefore the literature $\mathrm{CMC}$ (CAC) data obtained from the surface tension measurements were used for further analysis [1, 2, 25, 27].

Many authors suggest that short-chain alcohols behave in a somewhat similar way to classical surfactants both at the water-air interface and in the bulk phase of aqueous solution [2-6, 9]. It means that not only do they adsorb at that interface and reduce the solvent surface tension but they also tend to aggregate in the solution over a certain concentration.

If the alcohol behaviour at the water-air interface is similar to that of surfactants, there should be a relation between the efficiency of adsorption of these groups of compounds. It is known that the adsorption efficiency may be expressed by the standard Gibbs free energy of adsorption $\left(\Delta G_{a d s}^{0}\right)[1]$. 


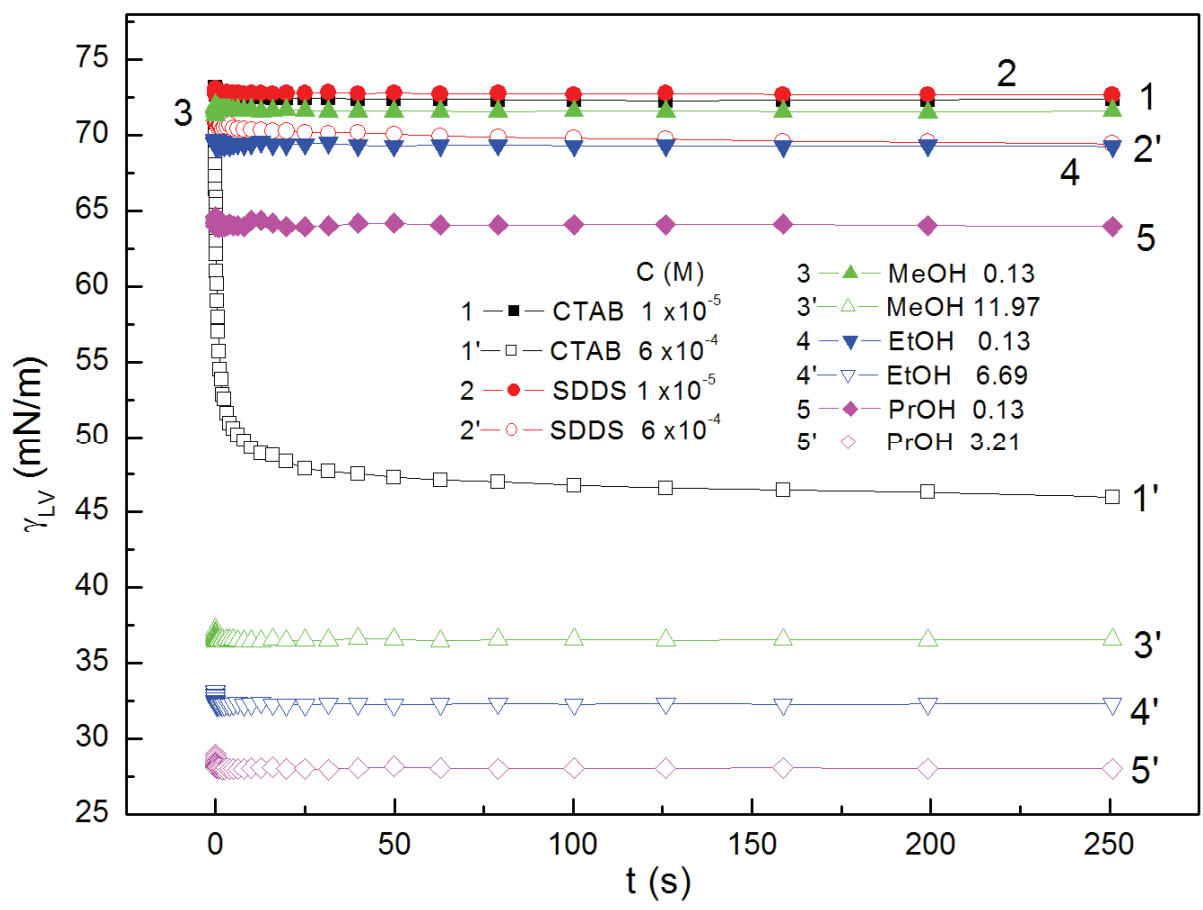

Fig. 1. Dependence between dynamic surface tension of the solution $\left(\gamma_{L V}\right)$ and the surface age $(t)$. Curves $1,1^{\prime} ; 2,2^{\prime} ; 3,3^{\prime} ; 4,4^{\prime}$ and 5, 5' correspond to the aqueous solutions of CTAB, SDDS, methanol, ethanol and propanol at a proper concentration, respectively.

In the case of surfactants this energy depends on the length of the hydrocarbon chain of their molecules. On the other hand, there is a correlation between $\Delta G_{a d s}^{0}$ and the number of $-\mathrm{CH}_{2}-$ groups $(n)$. Our earlier studies have shown that the $-\mathrm{CH}_{3}$ group can be treated as two $\mathrm{CH}_{2}-$ ones [26, 27]. Taking this fact into account, the dependence between $\Delta G_{a d s}^{0}$ of alcohols (taken from our previous paper [2]) and $(n+1)$ was analyzed. It proved that this dependence is linear and fulfills the following expression (Fig. 2a):

$$
\Delta G_{a d s}^{0}=1.047-3.9(n+1)
$$




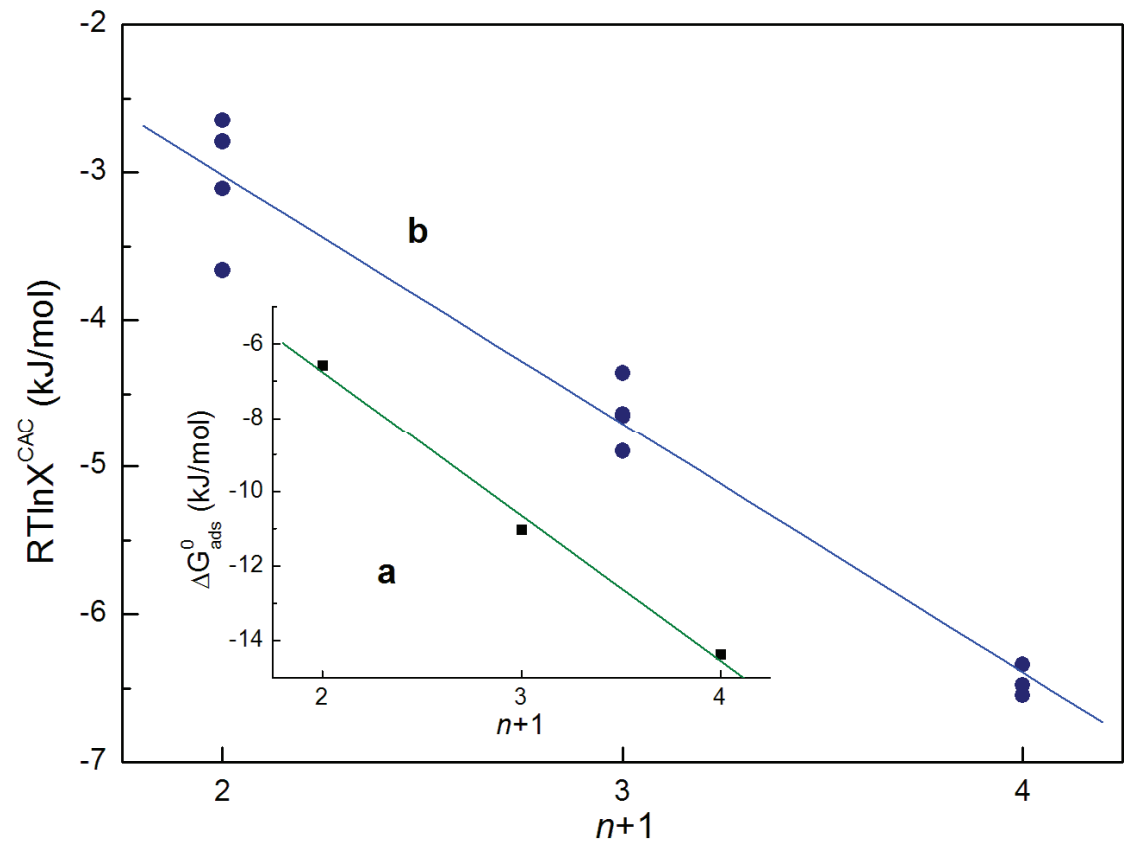

Fig. 2. a - Dependence between standard free energy of short-chain alcohol adsorption $\left(\Delta G_{a d s}^{0}\right)$ at the water-air interface and $n+1(n-$ number of carbon atoms in surfactant hydrophobic chain) [2].

$\mathrm{b}$ - Dependence between chemical potential of mixing of short-chain alcohols $\left(R T \ln X^{C A C}\right)$ and $n+1$ [2]. Points correspond to the CAC values obtained from surface tension, density and viscosity measurements [2].

Next we used Eq. (1) to calculate $\Delta G_{a d s}^{0}$ for such surfactants as sodium dodecylsulfate (SDDS), hexadecyltrimethylammonium bromide (CTAB), hexadecylpyridinium bromide (CPyB) and tetradecyltrimethylammonium bromide (TTAB) and compared these values with the literature data (Fig. 3) [25]. As follows from these calculations, it is possible to predict the $\Delta G_{a d s}^{0}$ for surfactants for which $n$ is equal to 12,14 and 16 on the basis of Eq. (1). It means that adsorption behaviour of surface active agents depends more on the chain length than on the kind of hydrophilic group and that the adsorption behaviour of methanol, ethanol and propanol is nearly the same as for classical surfactants.

On the surface tension isotherms of alcohols and surfactants, characteristic inflection points are observed (Fig. 4) [2, 25]. 


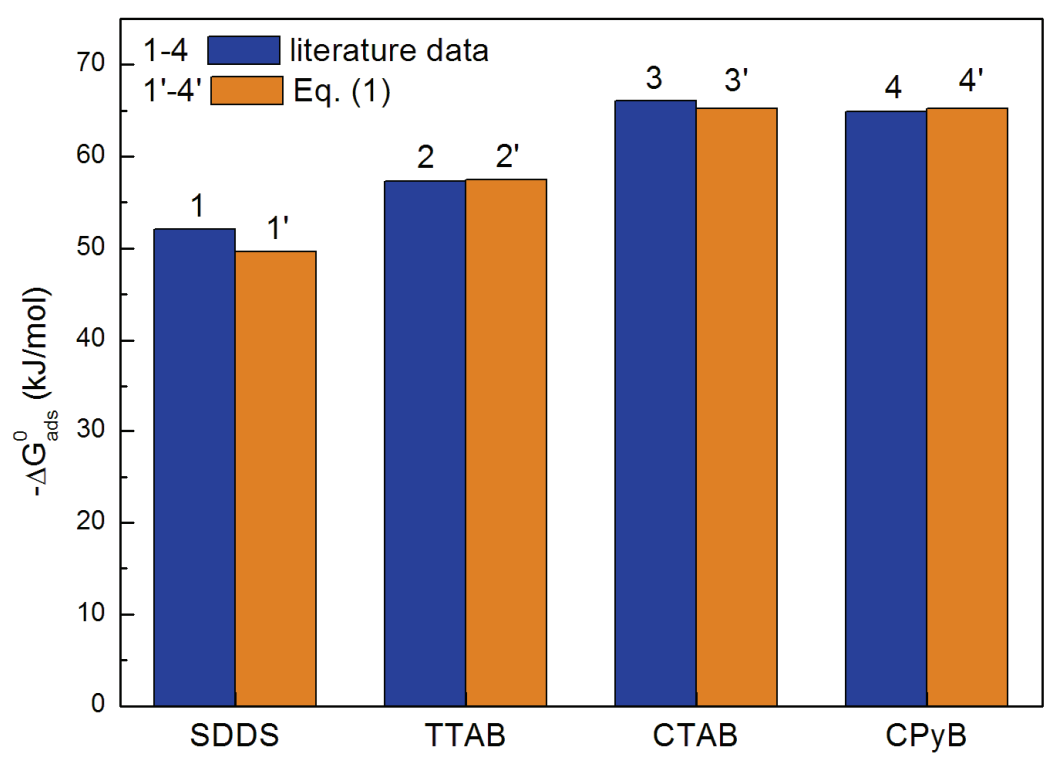

Fig. 3. Comparison of the standard free energy of surfactant adsorption $\left(\Delta G_{a d s}^{0}\right)$ at the water-air interface calculated from Eq. (1) (curves 1'-4') and the literature data (curves 1-4) [25]. Curves 1, 1'; 2, 2'; 3, 3'; 4, 4' and 5, 5' correspond to the aqueous solutions of SDDS, TTAB, CTAB and CPyB, respectively.

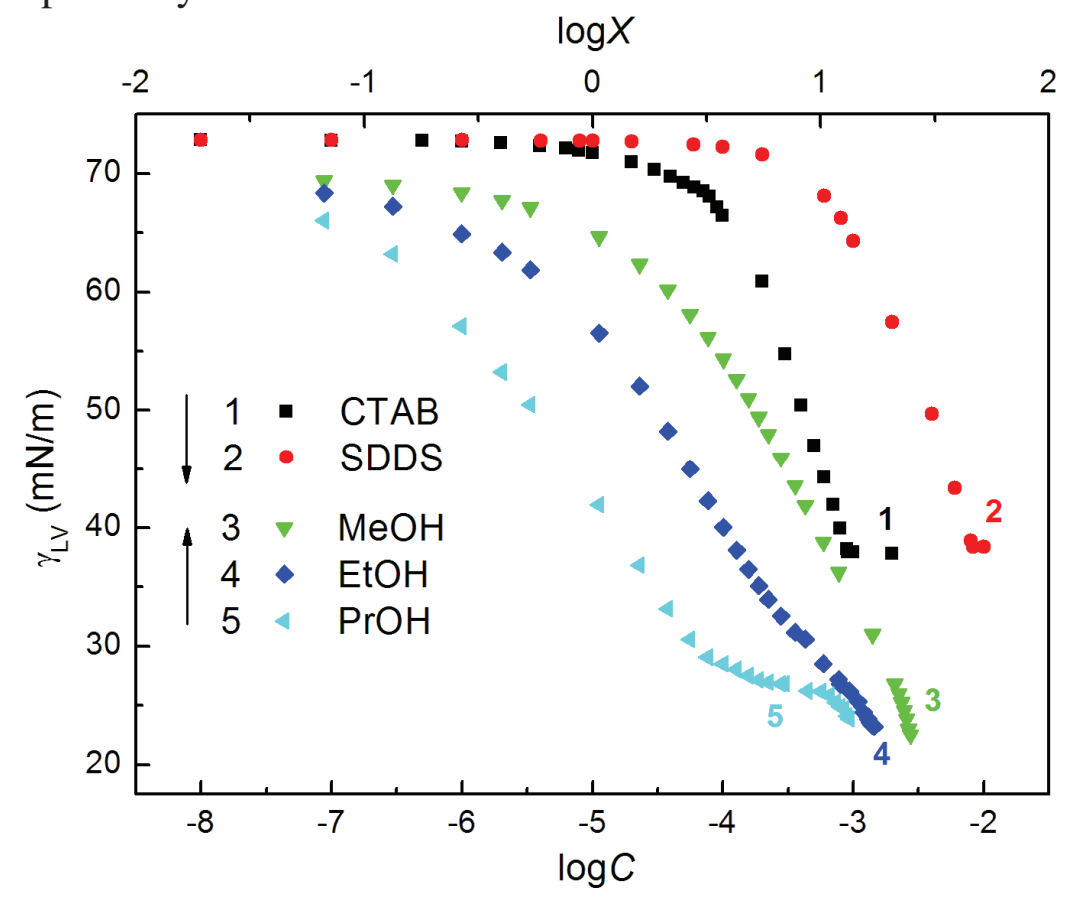

Fig. 4. Surface tension $\left(\gamma_{L V}\right)$ isotherm of the solution vs. logarithm of the concentration of surfactant (curves 1,2) and alcohol (curves 3-5) in the bulk phase $[2,25]$. Curves $1-5$ correspond to the aqueous solutions of CTAB, SDDS, methanol, ethanol and propanol, respectively. 
These points, which can be related to the aggregation of solute molecules in the bulk phase are less visible for alcohols. Based on the surface tension, viscosity and density measurements of the aqueous solutions of methanol, ethanol and propanol, our previous studies [2] indicate clearly that at the proper alcohol concentration, its molecules start to self-associate. The mole fraction values corresponding to the critical aggregation concentration (CAC) of ethanol and propanol determined by us at $293 \mathrm{~K}$ were close to those obtained at $298 \mathrm{~K}$ by Kahlweit et al. [5], but lower than those determined by Zana and Eljebari [6], and Hayashi and Udagawa [9]. In the case of methanol, its mole fraction corresponding to its CAC was considerably lower than that determined by Zana and Eljebari [6]. To confirm the aggregation of alcohol molecules in their aqueous solutions, the size of these aggregates was determined by the dynamic light scattering measurements. It occurred that propanol forms the largest aggregates of these three alcohols, and methanol forms mainly trimers [2]. It should be also mentioned that our studies dealing with the volumetric properties of some surfactants and their mixtures in the presence of short-chain alcohols [1822] showed that in such systems aggregation of alcohol molecules takes place too and the CAC values practically do not depend on the concentration of surfactants and composition of their mixture.

It is interesting if such relationship exists between the CAC of alcohols and the CMC of surfactants. Based on the experimental data for straight-chain ionic surfactants Klevens [24] found the following expression:

$$
\log C M C=A-B n
$$

where $A$ is a constant for a particular ionic head at a given temperature and $B$ is a constant that has approximately the same value for all mentioned homologous series of surfactants connected with the interactions of their hydrophobic parts through water.

According to the thermodynamic rules [35], behaviour of a given solution component can be better characterized by its chemical potential than the $\log C M C$. As it is commonly known, the chemical potential of a given component is equal to the sum of the standard and mixing potential [35]. The mixing potential is expressed by $R T \ln a$ where $a$ is the activity of a given component. If the activity is expressed by the asymmetric definition (if the mole fraction of a given solute approaches 0 , its activity coefficient approaches 1), then for the dilute solutions of surface active agents it can be written that the chemical potential of 
mixing is equal to $R T \ln X$ where $X$ is the mole fraction of a surface active agent.

The chemical potential of mixing is the partial molar property. Thus the chemical potential of straight-chain surface active agents in the homologous series at their critical concentration of aggregation should depend on the number of carbon atoms in their molecules. Therefore the dependence between the chemical potential of alcohol mixing and $(n+1)$ was analyzed. For this analysis the CAC data obtained from the surface tension, density and viscosity isotherms were taken [2].

It was found that there is a linear dependence between $R T \ln X^{C A C}$ and $(n+1)$ which fulfills the equation (Fig. $2 b)$ :

$$
R T \ln X^{C A C}=0.355-1.687(n+1)
$$

Taking Eq. (3) into account, the chemical potential of mixing of the homologous series of straight-chain alkyl sulfates, alkyl sulfonates, alkyl ammonium chlorides, alkyl trimethylammonium bromides, and alkyl pyridinium bromides in the range of $n$ from 8 to 16 was calculated (Fig. 5, line 1) [2]. This potential was compared to that obtained on the basis of their CMCs [1, 26, 27] (Fig. 5, points 2-6). For the determination of the chemical potential of surfactant mixing, the expression $R T \ln X^{C M C}$ was applied (where $X^{C M C}$ is the mole fraction of surfactant at the CMC), which was calculated from the following expression:

$$
X^{C M C}=\frac{C M C}{\omega}
$$

where $\omega$ is the number of water moles in $1 \mathrm{dm}^{3}$, which is equal to 55.4 at $293 \mathrm{~K}$.

It turned out that the chemical potential of mixing of alkyl sulfates is nearly the same as that predicted on the basis of the CAC of alcohols (Fig. 5, points 2) [2, 27]. There are some differences for other homologous series of surfactants, but this dependence is linear for all studied homologous series at nearly the same slope. It means that the chemical potential of mixing at the concentration at which the surface active agents start to form aggregates in the bulk phase depends on the number of carbon atoms in the straight hydrophobic chain and the influence of the kind of hydrophilic group in the surfactant molecule is not considerable. 


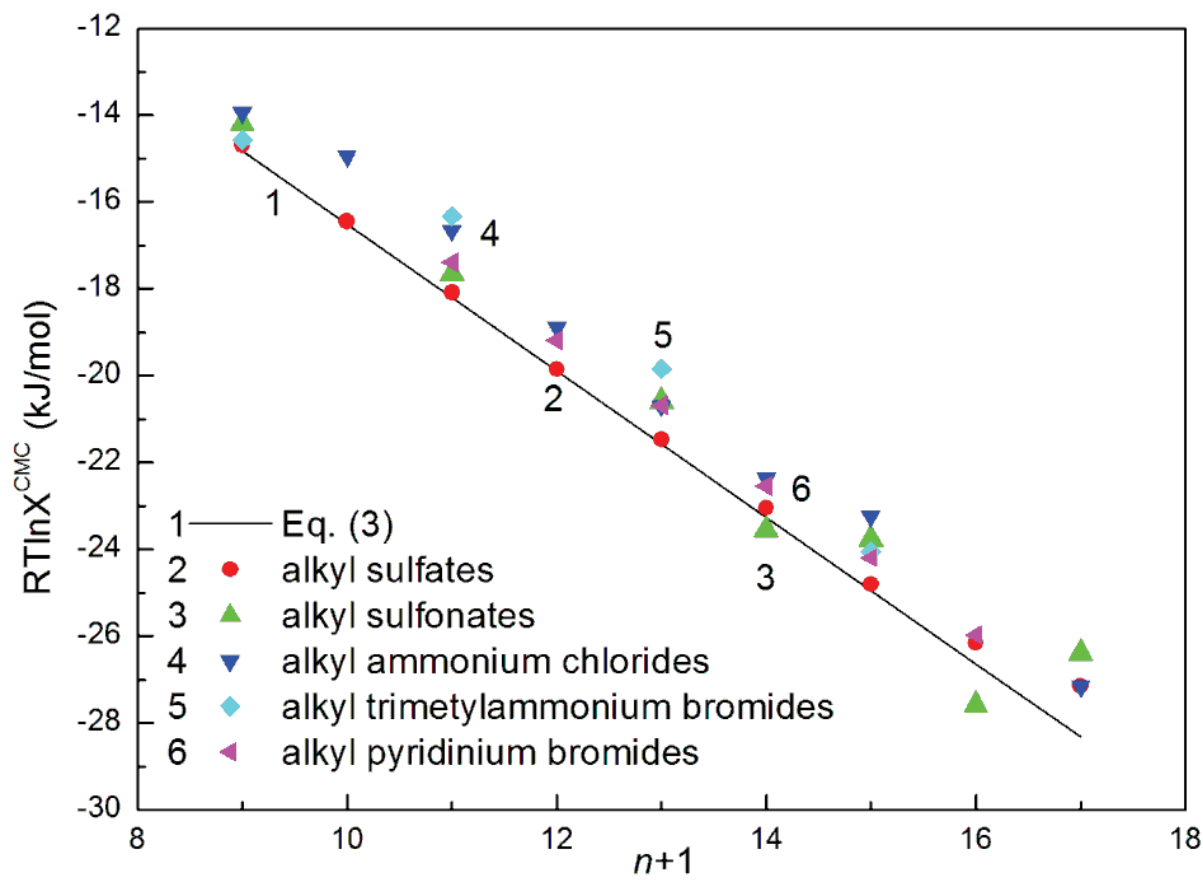

Fig. 5. Dependence between the chemical potential of mixing of surfactants $\left(R T \ln X^{C M C}\right)$ and $n+1$. Curve 1 corresponds to $R T \ln X^{C M C}$ values calculated from Eq. (3); points 2-6 correspond to $R T \ln X^{C M C}$ obtained from literature data $[1,26,27]$.

\section{CONCLUSIONS}

From our experiments and the thermodynamic considerations of the literature data dealing with the surface and volumetric properties of shortchain alcohols and some classical surfactants the following conclusions can be drawn:

The behaviour of short-chain alcohols at the water-air interface is similar to that of the classical surfactants. On the basis of the standard Gibbs free energy of adsorption, it is possible to predict this energy for the straight-chain ionic surfactants. It means that the adsorption efficiency of the surface active agents at the water-air interface depends only on the length of the straight-chain hydrophobic groups. The chemical potential of straight-chain surface active agents at the CMC depends linearly on the number of carbon atoms increased by 1 in the hydrophobic chains of these compounds. On the basis of the chemical potential of mixing of short-chain alcohols at their CAC, it is possible to predict the chemical potential of mixing of the straight-chain ionic surfactants at the CMC. It 
means that the there is only insignificant influence of the kind of hydrophilic group on the chemical potential of surfactant mixing at the $\mathrm{CMC}$ and that the behaviour of alcohols in the bulk phase of aqueous solutions is similar to those of straight-chain ionic surfactants.

\section{REFERENCES}

[1] M.J. Rosen, Surfactants and Interfacial Phenomena, WileyInterscience, New York, 2004.

[2] A. Chodzińska, A. Zdziennicka and B. Jańczuk, J. Sol. Chem., 41, 2226, (2012).

[3] R. Zana, Adv. Colloid Interface Sci., 57, 1, (1995).

[4] Y.F. Yano, J. Colloid Interface Sci., 284, 255, (2005).

[5] M. Kahlweit, G. Busse and J. Jen, J. Phys. Chem., 95, 5580, (1991).

[6] R. Zana and M.J. Eljebari, J. Phys. Chem., 97, 11134, (1993).

[7] K. Yoshida and T. Yamaguchi, Z. Naturforsch., 56a, 529, (2001).

[8] A.B. Roney, B. Space, E.W. Castner, R.L. Napoleon and R.B. Moore, J. Phys. Chem. B, 108, 7389, (2004).

[9] H. Hayashi and Y. Udagawa, Bull. Chem. Soc. Jpn., 65, 155, (1992).

[10] A. Vrhovšek, O. Gereben, A. Jamnik and L. Pusztaj, J. Phys. Chem. B, 115, 13473, (2011).

[11] M. Tomsic, A. Jamnik, G. Fritz-Popovski, O. Glatter and L. Vlcek, J. Phys. Chem. B, 111, 1738, (2007).

[12] I. Akiyama, M. Ogawa, K. Takase, T. Takamuku, T. Yamaguchi and N. Ohtori, J. Sol. Chem., 33, 797, (2004).

[13] K. Lin, X. Zhou, Y. Luo and S. Liu, J. Phys. Chem. B, 114, 3567, (2010).

[14] S. Dixit, J. Crain, W. C.K. Poon, J.L. Finney and A.K. Soper, Nature, 416, 829, (2002.

[15] J. Fidler, P.M. Rodger, J. Phys. Chem. B, 103, 7695, (1999).

[16] S. Alavi, S. Takeya, R. Ohmura, T.K. Woo and J.A. Ripmeester, J. Chem. Phys., 133, 074501-1, (2010).

[17] J.E. Desnoyers, D. Hetu and G. Perron, J. Sol. Chem., 12, 427, (1983).

[18] M. Bielawska, A. Chodzińska, B. Jańczuk and A. Zdziennicka, Colloids Surf. A, 424, 81, (2013). 
[19] M. Bielawska, B. Jańczuk and A. Zdziennicka, J. Surfactants Deterg., 16, 203, (2013).

[20] M. Bielawska, B. Jańczuk and A. Zdziennicka, Colloids Surf. A, 454, 65, (2014).

[21] M. Bielawska, B. Jańczuk and A. Zdziennicka, Colloids Surf. A, 464, 57, (2015).

[22] M. Bielawska, B. Jańczuk and A. Zdziennicka, Colloids Surf. A, (2015), in press, doi:10.1016/j.colsurfa.2014.12.047.

[23] A.I. Vogel, Preparatyka organiczna, WNT, Warszawa, 2006 (in Polish).

[24] H.B. Klevens, J. Am. Oil Chem. Soc., 30, 74, (1953).

[25] A. Zdziennicka, K. Szymczyk, J. Krawczyk and B. Jańczuk, Fluid Phase Equilib., 318, 25, (2012).

[26] A. Zdziennicka, K. Szymczyk, J. Krawczyk and B. Jańczuk, Fluid Phase Equilib., 322-323, 126, (2012).

[27] B. Jańczuk, M.L. Gonzalez-Martin, A. Zdziennicka and W. Wójcik, Tenside Surfact. Det., 39, 28, (2002).

[28] M. Czerniawski, Roczniki Chem., 40, 1265, (1966) (in Polish).

[29] P.H. Elworthy and K.J. Mysels, J. Colloid Interface Sci., 21, 331, (1966).

[30] J.M. del Rio, G. Prieto, F. Sarrniento and V. Mosquera, Langmuir, 11, 1511 (1995).

[31] E. Fisicaro, M. Biemmi, C. Compari, E. Duce and M. Peroni, J. Colloid Interface Sci., 305, 301, (2007).

[32] E. Rodenas, C. Dolcet, M. Valiente and E.C. Valeron, Langmuir, 10, 2088 (1994).

[33] [33] M.H. Ropers, G. Czichocki and G. Brezesinski, J. Phys. Chem. B, 107, 5281 (2003).

[34] [34] T.M. Perger and M. Bešter-Rogač, J. Colloid Interface Sci., 313, 288, (2007).

[35] [35] P. Atkins and J. de Paula, Physical Chemistry, Oxford University Press, Oxford, 2009. 


\section{CURRICULA VITAE}

Magdalena Bielawska is a Ph. D. student in the Department of Interfacial Phenomena (Faculty of Chemistry, Maria Curie-Sklodowska University in Lublin, Poland). Her scientific interest is focused on wettability of solids strictly connected with surface and volumetric properties of surfactants and their mixtures with short-chain alcohols. She is a co-author of 9 papers.

Anna Zdziennicka graduated from Maria Curie-Sklodowska University in Lublin (1988, Faculty of Mathematics, Physics and Chemistry). Received her Ph. D. degree in physical chemistry from UMCS. She works in the Department of Interfacial Phenomena in the Faculty of Chemistry, UMCS. Her research work is largely concentrated on physicochemistry of the surface and interfacial phenomena. She is a co-author of 90 papers.

Bronistaw Jańczuk is a professor at the Department of Interfacial Phenomena, Faculty of Chemistry UMCS Lublin, Poland, where he obtained all his scientific degrees (Ph. D. 1973, Habilitation 1985, Professor 1991). Since 2001 he has been the head of the Department of Interfacial Phenomena. He has published over 310 original papers in professional journals and books. His research areas are interfacial phenomena such as adsorption, wettability and adhesion in a wide range of systems including such solids as minerals, polymers, clay minerals, constituents of soil, and organic substances, particularly properties of surfactants and their mixtures. 